\section{Transition to Members in Action e-news}

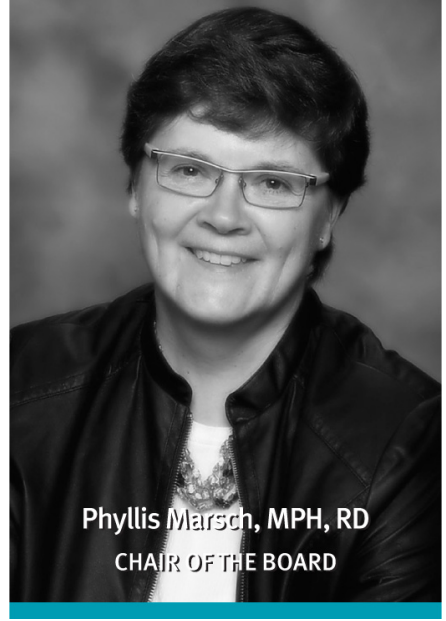

Chair's Message

Message de la Présidente

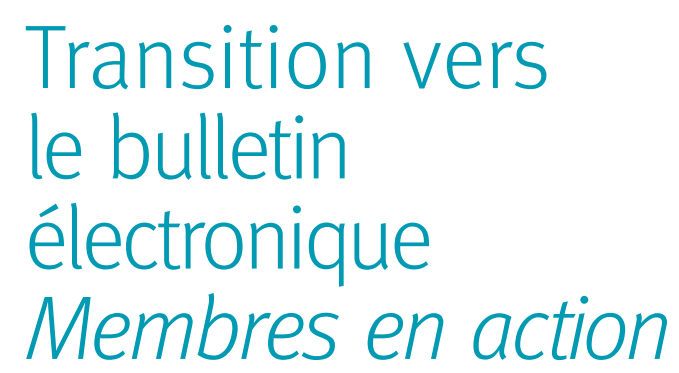

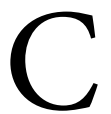

ompleting my two-year term as Board Chair draws me to reflect upon the past five years as a Board member and to recognize that this is a time of transition.

With this transition, comes a shift of the Chair's Message from the Canadian Journal of Dietetic Practice and Research to Members in Action (MIA) e-news, since the Chair's Message is written to members and MIA is the member communications vehicle. The message will appear twice a year and is not intended to replace the "From the Board" articles. This is therefore the final Chair's Message in the Journal and first time in MIA.

In reflection, there have been many highlights during my term on the Board. If pressed to choose one, it would be the joint work that was completed by the DC Management Team and Board in the development of a set of new results the organization is to producecalled Ends. As a basis of environmental scanning, key drivers were identified, and data including the Board facilitated member/ nonmember survey (totalling over 2,000 respondents) were analyzed. As a further outcome, the process of shaping the preferred future context within which DC operates was acknowledged.

It has been a milestone in my career, to serve on the DC Board. Second to none, it has been an honour working with YOU as members - the owners of DC, the dedicated DC staff and CEO Marsha Sharp, and Board colleagues who volunteer countless hours to ensure the health of the organization. It remains without a doubt that together we contribute immeasurable expertise and a definite passion for advancing our profession.

(Can J Diet Pract Res. 2017;78:51)

(DOI: 10.3148/cjdpr-2017-017)

Published at dcjournal.ca on 24 May 2017
$\mathrm{M}$ on mandat de deux ans en tant que présidente du conseil d'administration touchant à sa fin, j'en suis venue à réfléchir à mes cinq dernières années au sein du conseil. Or, je constate qu'une période de transition s'opère.

Avec cette transition, un changement s'annonce. Le message de la présidente de la Revue canadienne de la pratique et de la recherche en diététique, qui s'adresse aux membres, sera désormais publié dans le bulletin électronique Membres en action, car il s'agit du moyen privilégié pour communiquer avec les membres des DC. Ce message, qui sera publié deux fois par année, ne vise pas à remplacer les articles de la rubrique "De votre conseil ». Il s'agit donc du dernier message de la présidente dans la Revue et du premier dans le bulletin électronique Membres en action.

Il me semble qu'avec le recul, plusieurs faits saillants ont ponctué mon mandat au conseil d'administration. Si l'on me demandait de choisir le plus marquant, je pencherais pour le travail conjoint réalisé par l'équipe de gestion et le conseil d'administration des DC en vue d'élaborer l'ensemble de nouveaux résultats que doit produire l'organisation, à savoir nos objectifs. Pour ce faire, nous avons réalisé une analyse environnementale en identifiant des facteurs clés et en analysant des données, dont celles issues du sondage réalisé par le conseil d'administration auprès des membres et des non-membres (plus de 2000 répondants). En outre, le processus qui consiste à façonner le contexte privilégié dans lequel les DC exerceront leurs activités à l'avenir a été confirmé.

Le fait d'avoir siégé au conseil d'administration des DC est certes un jalon dans ma carrière. Par ailleurs, cela a été un honneur de travailler avec vous, les membres, véritables propriétaires des DC, ainsi qu'avec le personnel dévoué et Marsha Sharp, notre directrice générale et, bien sûr, les collègues du conseil, qui ont consacré bénévolement d'innombrables heures pour veiller à la santé de l'organisation. Sans l'ombre d'un doute, ensemble, nous mettons notre incontestable expertise et notre vive passion au service de l'avancement de notre profession.

(Rev can prat rech diétét. 2017; 78:51)

(DOI: $10.3148 /$ cjdpr-2017-017)

Publié au dcjournal.ca le 24 mai 2017

\begin{tabular}{ll}
$\begin{array}{ll}\text { 2016-2017 } \\
\text { Board of } \\
\text { Directors }\end{array}$ & $\begin{array}{l}\text { Phyllis Marsch (Chair) } \\
\text { Winnipeg, MB } \\
\text { 204-275-1918 }\end{array}$ \\
\cline { 1 - 1 } $\begin{array}{l}\text { Conseil } \\
\text { d'administration }\end{array}$ & $\begin{array}{l}\text { Doug Cook } \\
\text { Toronto, ON } \\
416-413-9095\end{array}$
\end{tabular}

Colinda Hunter Edmonton, $\mathrm{AB}$ 780-577-3333

Sue Conlan Halifax, NS 902-697-2157
Nicole Spencer

Vancouver, BC 604-564-6534

Joane Routhier Kirkland, QC 514-398-7749

\author{
Judy Sheeshka \\ Surrey, BC \\ 778-294-3723 \\ Melissa Baker \\ Toronto, ON \\ 647-881-4576
}

Jean Helps Headingly, MB 204-837-3166

Chief Executive Officer Marsha Sharp 416-642-9314 